\title{
Prediction of paired-associate latencies after the last error by an all-or-none learning model
}

John Theios UNIVERSITY OF TEXAS

\begin{abstract}
Abstraet
An all-or-none learning model is presented which makes predictions for response latencies in pairedassociate learning. The predicted latencies decrease on trials following the last error of a criterion run and accurately describe the latency data of an experiment by Peterson (1965).
\end{abstract}

\section{Problem}

Peterson (1965) has stated that "For an incremental view of learning, the latency of response would be expected to decrease after the $\mathrm{S}$ stops making errors. However, decreasing latencies after errors cease are difficult to incorporate into an all-or-none model of learning." Peterson (1965) gave 15 undergraduates 20 trials to learn 10 paired-associates. The stimuli were 10 consonant bigrams, and the responses were the digits 1 to 8 . He found that the group mean latency decreased on the 10 trials following an S's last error. He interpreted the data as indicating that "Speed of occurence (of the correct response) appears to be a measure of strength of an association which changes gradually over trials."

The question of crucial importance to learning theory is not whether learning is strictly "'all-or-none." Rather it is whether learning is grossly discrete or approximately continuous. A grossly discrete process may have a number of individual mechanisms, each strictly allor-none. When cast in these terms, the Peterson (1965) data are not at all at variance with a discrete, all-ornone position on learning.

As a case in point, suppose that a complete stimulusresponse association is formed strictly on one trial. Behavioristically, an association is defined as the occurrence of a particular response to a presented stimulus. Assume further that the association is first stored in an imperfect, temporary memory and later transferred to a perfect, relatively permanent memory. Then response latency would be a direct function of retrieval time or memory search time, and it would not be unreasonable to expect that retrieval of the association from temporary memory would take longer than retrieval from permanent memory. These notions lead to an all-or-none learning model which may be defined by the following explicit assumptions:

1. The response given on a trial to a particular stimulus item depends only on the state of conditioning of the individual stimulus-response association. The four possible states are: no association (N), association stored in temporary memory $(\mathrm{T})$, association temporarily forgotten or lost from memory (F), and association stored in permanent memory (P).

2. The $S$ makes an error after each presentation of the stimulus if the correct association has not been formed.

3. A correct association may be formed and stored in temporary memory with a fixedprobability c following information about the correct response, i.e. following reinforcement.

4. With probability g, equal to the reciprocal of the number of possible response alternatives, the correct association may exist at the start of the experiment and already be stored in temporary memory.

5. An association may be transferred from temporary memory to permanent memory with a fixed probability $s$ following a reinforced correct response. If the association is not transferred to permanent memory it may be lost from temporary memory with a fixed probability q during the intertrial interval.

6. Following information about the correct response, a forgotten association is reformed and restored in temporary memory with probability 1.0. However, during the intertrial interval the reformed association may be lost from temporary memory with probability $q$.

7. In each state of conditioning $i,(i=N, F, T, P)$ there is a probability distribution $d(i)$ of response times each of which has a mean $L_{i}$, such that $L_{T}$ is greater than $\mathrm{L}_{\mathrm{p}}$.

The model is a special case of a general model proposed by Bower \& Theios (1964) and is completely specified by the following state transition matrix, response probability vector, mean latency vector, and starting state vector,

$$
\text { P } \quad \mathrm{T} \quad \mathrm{F} \quad \mathrm{N} \text { Pr(error) latency } \operatorname{Pr}(\text { start })
$$

$\mathrm{P}\left[\begin{array}{cccc}1 & 0 & 0 & 0 \\ \mathrm{~T} & \mathrm{~s} & (1-\mathrm{s}) \mathrm{p}(1-\mathrm{s}) \mathrm{q} & 0 \\ 0 & \mathrm{p} & \mathrm{q} & 0 \\ 0 & \mathrm{cp} & \mathrm{cq} & 1-\mathrm{c}\end{array}\right]\left[\begin{array}{l}0 \\ 0 \\ 1 \\ 1\end{array}\right]\left[\begin{array}{c}\mathrm{L}_{\mathrm{P}} \\ \mathrm{L}_{\mathrm{T}} \\ \mathrm{L}_{\mathrm{F}} \\ \mathrm{L}_{\mathrm{N}}\end{array}\right]\left[\begin{array}{c}0 \\ \mathrm{~g} \\ 0 \\ 1-\mathrm{g}\end{array}\right]$

where $p=(1-q)$. The parameter $g$ represents the probability of a correct guess on the first trial of the experiment. The rows of the transition matrix representpossible states of an association on any trial $n$ of an experiment, and the columns represent possible states on trial $n+1$. The cells of the matrix give the probability 
of going from one given state to another during the intertrial interval.

The model describes a learning process in which response probability changes in one strictly all-or-none step, zero to unity or unity to zero. The mean response latency also takes only one increment if $\mathrm{L}_{\mathrm{N}}, \mathrm{L}_{\mathrm{T}}$, and $\mathrm{L}_{\mathrm{F}}$ are all equal. On the basis of latency data published in Millward's (1964b) Fig. 4, there is reason to assume that the three mean latencies are equal for some experimental situations.

In order to make predictions, let $\mathrm{W}_{\mathrm{T}, \mathrm{n}}$ represent the probability that the association is stored in temporary memory at the beginning of the trial of the $n$-th correct response of the criterion run. Likewise, let $\mathrm{W}_{\mathrm{P}, \mathrm{n}}$ represent the probability that it is stored in permanent memory by trial $\mathrm{n}$ of the criterion run. According to the model,

and

$$
\begin{aligned}
& \mathrm{W}_{\mathrm{T}, \mathrm{n}}=[\mathrm{p}(1-\mathrm{s})]^{\mathrm{n}-1} \\
& \mathrm{~W}_{\mathrm{P}, \mathrm{n}}=1-[\mathrm{p}(1-\mathrm{s})]^{\mathrm{n}-1} .
\end{aligned}
$$

Equations 1 and 2 state that the first correct response of a criterion run occurs when the association is stored in temporary memory, but that after a large number of consecutive correct responses the association will have been transferred to permanent memory. The predicted average group latency on trial $\mathrm{n}$ of the criterion run will be given by

$$
\mathrm{L}_{\mathrm{n}}=\left(\mathrm{L}_{\mathrm{T}}\right)\left(\mathrm{W}_{\mathrm{T}, \mathrm{n}}\right)+\left(\mathrm{L}_{\mathrm{P}}\right)\left(\mathrm{W}_{\mathrm{p}, \mathrm{n}}\right) .
$$

Expressions equal to or similar to Equation 3 also follow from other all-or-none learning models developed by Atkinson (1962), Crothers (1963), Millward (1964a), and Suppes \& Ginsberg (1963).

The purpose of this paper is to show that Equation 3 from the all-or-none learning models can accurately describe the latency data published by Peterson (1965).

\section{Nethod}

Since the model requires that the first response after the last error occur when the association is stored in temporary memory, an estimate of $\mathrm{L}_{\mathrm{T}}$ was obtained from Peterson's (1965) Fig. 1 as $1.9 \mathrm{sec}$., the mean latency on the first trial after the last error. Since the mean latencies were asymptotic after 10 consecutive correct responses, an estimate of $\mathrm{L}_{\mathrm{p}}$ was obtained as $.9 \mathrm{sec}$., the mean latency of the 10 -th response following the last error. Six estimates of the probability of $\mathrm{p}(1-\mathrm{s})$ were obtained by setting the observed mean latencies on criterion correct responses 3 to 8 equal to Equation 3 and solving for $\mathrm{p}(1-\mathrm{s})$. The mean of the 6 independent estimates, .6, was taken as the best estimate of $p(1-s)$.

\section{Results}

The mean latency on trials after the last error predicted by Equation 3 have been added to Peterson's (1965) curve which appears as Fig. 1. As can be seen, the all-or-none model is accurately predicting the data. Several other properties of the data also follow from the all-or-none model. The assumption that $\mathrm{L}_{\mathrm{N}}, \mathrm{L}_{\mathrm{T}}$, and $\mathrm{L}_{\mathrm{F}}$ are equal results in the prediction that there should be no reliable trends in latency prior to the last error. This was the case in the data. According to the model, the probability of a correct response on trials before the last error should increase from a value of $g$, in this case .125 , to a limit of $\mathrm{p}(1-\mathrm{s}) /(1-\mathrm{ps})$. The observed proportion of correct responses did increase on trials before the last error from .14 to .49 . Finally, the mean run of correct responses beginning on any trial before the last error should increase up to a limit as a function of the trial number. Runs of as long as six consecutive correct responses were observed in the data before the last error. According to the

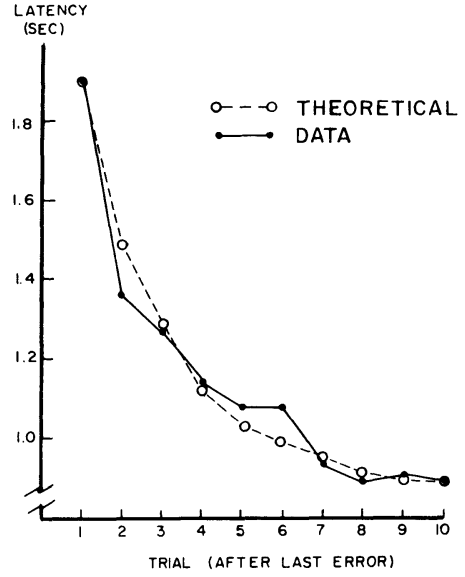

Fig. 1. Predicted and obtained mean latency for 10 trials after the last error in a paired-associate task (data from Peterson, 1965).

model, long runs like this may occur when the association is in temporary memory, especially if the parameters $s$ and $q$ are relatively small.

\section{Diseussion}

The model proposed here might be logically more plausible if permanent storage were permitted to take place after either an incorrect or a correct response, since $S$ receives the same information following each trial. This modification, however, would result in predictions which would differ very little, if any, from those that were made. The modified model has the undesirable property that at least one more parameter would have to be estimated from the data in order to predict the latencies after the last error.

The all-or-none latency model proposed by Millward (1964b) and tested and rejected by Peterson (1965) is a special case of the all-ornone latency model reported in this paper. The two models make identical predictions about the probability of a correct response if the parameter c equals 1.00 and p equals $g$. The two models make identical predictions about response latency if $\mathrm{L}_{\mathrm{N}}$ is equal to $\mathrm{L}_{\mathrm{T}}$, $\mathrm{L}_{\mathrm{N}}$ is equal to $\mathrm{L}_{\mathrm{F}}$, and $\mathrm{L}_{\mathrm{N}}$ is greater than $\mathrm{L}_{\mathrm{P}}$.

The implications of the present results are clear. Investigators should direct their research toward conducting experiments within the boundary conditions of one or more mathematically well defined models so that specific, unambiguous predictions about the data can be made. In most cases a model will not fit the data in all its aspects. The points where a model is not predicting the data are of prime importance scientifically, because they can indicate how the model can be improved. Through this process it is possible to obtain more and more general models. When experimental results are obtained which disconfirm an up-till-then adequate model, the model need not be completely rejected, but should become a special case of a better, more general model.

\section{Referenees}

ATKINSON, R. C. Choice behavior and monetary payoff: strong and weak conditioning. In J. H. Criswell, H. Solomon, \& P. Suppes (Eds.), Mathematical methods in small group processes. Stanford, Calif.: Stanford University Press, 1962. Pp. 23-34.

BOWER, G. H., \& THEIOS, J. A learning model for discrete performance levels. In R. C. Atkinson (Ed.), Studies in mathematical psychology. Stanford, Calif.: Stanford University Press, 1964. Pp. 1-31.

CROTHERS, E. General Markov models for learning with inter-trial forgetting. Tech. Rep. No. 53, Psychology Series, Institute for Mathematical Studies in the Social Sciences, Stanford University.

MILLWARD, R. An all-or-none model for noncorrection routines with elimination of incorrect responses. J. math. Psychol., 1964a, 1, 392-404.

MILLWARD, R. Latency in a modified paired-associate learning experiment. J. verbal Learn. verbal Behav., 1964b, 3, 309-316.

PETERSON, L. R. Paired-associate latencies after the last error. Psychon. Sci., 1965, 2, 167-168.

SUPPES, P., \& GINSBERG, R. A fundamental property of all-or-none models. Psychol. Rev., 1963, 70, 139-161. 